\title{
Parity nonconservation effect in resonance recombination of polarized electrons with heavy hydrogenlike ions
}

\author{
A. V. Maiorova, ${ }^{1}$ V. M. Shabaev, ${ }^{1}$ A. V. Volotka,,${ }^{1,2}$ \\ V. A. Zaytsev, ${ }^{1}$ G. Plunien, ${ }^{2}$ T. Stöhlker ${ }^{3,4,5}$ \\ ${ }^{1}$ Department of Physics, St.Petersburg State University, \\ Ulianovskaya 1, Petrodvorets, St.Petersburg 198504, Russia \\ ${ }^{2}$ Institut für Theoretische Physik, Technische Universität Dresden, \\ Mommsenstrasse 13, D-01062 Dresden, Germany \\ ${ }^{3}$ Gesellschaft für Schwerionenforschung, \\ Planckstrasse 1, D-64291 Darmstadt, Germany \\ ${ }^{4}$ Physikalisches Institut, Universität Heidelberg, \\ Philosophenweg 12, D-69120 Heidelberg, Germany \\ ${ }^{5}$ Helmholtz-Institut Jena, D-07r43 Jena, Germany
}

(Dated: November 21, 2018)

\begin{abstract}
Parity nonconservation (PNC) effect in recombination of a polarized electron with a heavy H-like ion in case of resonance with a doubly excited state of the corresponding He-like ion is studied. It is assumed that photons of the energy corresponding to the one-photon decay of the doubly excited state into the $2^{1} S_{0}$ or the $2^{3} P_{0}$ state are detected at a given angle with respect to the incident electron momentum. Calculations are performed for heliumlike thorium $(Z=90)$ and gadolinium $(Z=64)$, where the $2^{1} S_{0}$ and $2^{3} P_{0}$ levels are near to cross and, therefore, the PNC effect is strongly enhanced.

PACS numbers: 11.30.Er, 34.80.Lx
\end{abstract}




\section{INTRODUCTION}

Parity nonconservation (PNC) effects caused by the weak neutral-current interaction were extensively studied in neutral atomic systems [1 3]. Recent progress on the theory of the PNC effects in neutral atoms is mainly related to evaluation of the QED correction and a signifcant improvement of the accuracy of the electron-correlation contribution (see Refs. [4, 5] and references therein). The accuracy of the theoretical predictions for the PNC effects in neutral atoms is mainly limited by an uncertainty of the electron-correlation contribution. In contrast to that, in highly charged few-electron ions the electron-correlation contribution, being suppressed by a factor $1 / Z$ ( $Z$ is the nuclear charge number), can be calculated to a very high accuracy employing the $1 / Z$ perturbation theory. This provides very good prospects for investigations of the PNC effects with heavy few-electron ions.

PNC experiments with highly charged ions were first discussed in Ref. [6], where it was proposed to use the close opposite-parity levels in He-like ions at $Z \sim 6$ and $Z \sim 29$. Later, the PNC effects with heavy ions were considered by a number of authors [7-19]. Most of these studies exploited the near-degeneracy of the $2^{1} S_{0}$ and $2^{3} P_{0}$ levels in He-like ions at $Z \sim 64$ and $Z \sim 90$, where the PNC effect is strongly enhanced (see, e.g., Refs. [7, 13, 1519]). In particular, in our recent investigation [16] we evaluated the PNC effect on the cross section of the radiative recombination of an electron into the $2^{1} S_{0}$ and $2^{3} P_{0}$ states of He-like ions for two experimental scenarios. In the first scenario, the incident electron is polarized, while the H-like ion is unpolarized, and the photon polarization is not detected. In the second one, linearly polarized photons are detected in an experiment with unpolarized electrons and ions. The numerical results for both scenarios were obtained and optimum cases in which the effect is most pronounced were found.

In the present paper, we study the PNC effect on recombination of a polarized electron with unpolarized hydrogenlike thorium $(Z=90)$ and gadolinium $(Z=64)$ ions in case of resonance with a doubly excited state of the He-like ion. We consider a scenario in which photons of the energy equal to the difference between the initial energy (the ground state energy of the H-like ion and the incident electron energy) and the energy of the He-like ion in the $2^{1} S_{0}$ or the $2^{3} P_{0}$ state are detected at a given angle. As the intermediate doubly excited states, we consider $\left(2 s_{1 / 2} 2 p_{1 / 2}\right)_{1},\left(2 s_{1 / 2} 2 p_{3 / 2}\right)_{1},\left(2 p_{1 / 2} 2 p_{3 / 2}\right)_{1},\left(2 s_{1 / 2} 3 p_{1 / 2}\right)_{1}$, and $\left(2 s_{1 / 2} 3 p_{3 / 2}\right)_{1}$ states, where the PNC effect on the dielectronic recombination cross section should be most 
pronounced. This is due to the fact that for such states we can choose the magnetic dipole (M1) transition to the $2^{1} S_{0} \equiv\left(1 s_{1 / 2} 2 s_{1 / 2}\right)_{0}$ or the $2^{3} P_{0} \equiv\left(1 s_{1 / 2} 2 p_{1 / 2}\right)_{0}$ state, while the PNCmixing channel is the electric dipole (E1) transition. The values of the photon emission angle with respect to the incident electron momentum, which correspond to the maximum PNC effect, are evaluated.

Relativistic units $(\hbar=c=1)$ and the Heaviside charge unit $\left(\alpha=e^{2} /(4 \pi), e<0\right)$ are used throughout the paper.

\section{BASIC FORMULAS}

We consider recombination of an electron having asymptotic four-momentum $p_{i}=\left(\varepsilon_{i}, \mathbf{p}_{\mathbf{i}}\right)$ and polarization $\mu_{i}$ with a heavy $\mathrm{H}$-like ion in the ground $1 s$ state at $Z \approx 90$ or $Z \approx 64$. We choose the incident electron energy $\varepsilon_{i}$ to get the resonance with a doubly excited state $d$ of the He-like ion, $E_{1 s}+\varepsilon_{i}=E_{d}$, and assume that photons of the energy corresponding to the decay of this state to the $2^{1} S_{0}$ or the $2^{3} P_{0}$ state are detected in experiment. In the resonance approximation, the cross section of the process is the sum of the dielectronic recombination $(\mathrm{DR})$ and radiative recombination $(\mathrm{RR})$ cross sections and an interference term. In the scenario we consider the photon polarization is not measured. Then, neglecting the weak interaction, the differential cross section of the one-photon recombination into the $2^{1} S_{0}$ or the $2^{3} P_{0}$ final state, $\sigma \equiv d \sigma / d \Omega$, is given by [20, 21]

$$
\sigma=\frac{(2 \pi)^{4}}{v_{i}} \mathbf{k}_{\mathbf{f}}^{2} \sum_{\boldsymbol{\epsilon}_{f}}\left|\sum_{M_{d}} \tau_{\gamma_{f}, f ; d} \frac{1}{E_{i}-E_{d}+i \Gamma_{d} / 2}\left\langle\Psi_{d}|I| \Psi_{i}\right\rangle+\tau_{\gamma_{f}, f ; i}\right|^{2}
$$

Here $i, d$, and $f$ are the initial, intermediate, and final states, respectively. $E_{i}=E_{1 s}+\varepsilon_{i}$ is the energy of the initial state, $E_{d}$ and $\Gamma_{d}$ are the energy and the width of the intermediate doubly excited state $d, I$ is the operator of the interelectronic interaction, $\tau_{\gamma_{f}, f ; i}$ is the RR amplitude into the final state $f, \tau_{\gamma_{f}, f ; d}$ is the transition amplitude from the intermediate state $d$ to the final state, $\mathbf{k}_{\mathbf{f}}$ is the photon momentum, and $v_{i}$ is the incident electron velocity.

For heavy few-electron ions we can generally use the one-electron approximation for the wave functions, since the interelectronic-interaction effects are suppressed by a factor $1 / Z$, compared to the interaction of the electrons with Coulomb field of the nucleus. Then the 
wave function of the initial state is

$$
\Psi_{i}\left(\mathbf{x}_{\mathbf{1}}, \mathbf{x}_{\mathbf{2}}\right)=\frac{1}{\sqrt{2}} \sum_{\mathcal{P}}(-1)^{\mathcal{P}} \mathcal{P} \psi_{1 s}\left(\mathbf{x}_{\mathbf{1}}\right) \psi_{p_{i} \mu_{i}}\left(\mathbf{x}_{\mathbf{2}}\right)
$$

where $\psi_{1 s}(\mathbf{x})$ is the one-electron 1s Dirac wave function, $\psi_{p_{i} \mu_{i}}$ is the incident electron Dirac wave function, $(-1)^{\mathcal{P}}$ is the parity of the permutation, and $\mathcal{P}$ is the permutation operator. Assuming that the electron momentum $\mathbf{p}_{\mathbf{i}}$ is directed along the quantization axis (we consider the process in the ion rest frame), the wave function of the incident electron can be expanded as

$$
\psi_{p_{i} \mu_{i}}(\mathbf{x})=\frac{1}{\sqrt{4 \pi}} \frac{1}{\sqrt{p_{i} \varepsilon_{i}}} \sum_{\kappa} \mathrm{i}^{l} \exp \left(i \Delta_{\kappa}\right) \sqrt{2 l+1} C_{l 0, \frac{1}{2} \mu_{i}}^{j \mu_{i}} \psi_{\varepsilon_{i} \kappa \mu_{i}}(\mathbf{x})
$$

where $\Delta_{\kappa}$ is the Coulomb phase shift, $C_{l 0, \frac{1}{2} \mu_{i}}^{j \mu_{i}}$ is the Clebsch-Gordan coefficient, and $\psi_{\varepsilon_{i} \kappa \mu_{i}}(\mathbf{x})$ is the partial electron wave with the Dirac quantum number $\kappa=(-1)^{j+l+1 / 2}(j+1 / 2)$ determined by angular momentum $j$ and parity of the state $l$.

If we neglect the weak electron-nucleus interaction, the wave functions of the $d$ and $f$ states can be written as

$$
\Psi_{J M}\left(\mathbf{x}_{\mathbf{1}}, \mathbf{x}_{\mathbf{2}}\right)=A_{N} \sum_{m_{1} m_{2}} \sum_{\mathcal{P}}(-1)^{\mathcal{P}} \mathcal{P} C_{j_{1} m_{1}, j_{2} m_{2}}^{J M} \psi_{j_{1} m_{1}}\left(\mathbf{x}_{\mathbf{1}}\right) \psi_{j_{2} m_{2}}\left(\mathbf{x}_{\mathbf{2}}\right)
$$

where $\psi_{j m}(\mathbf{x})$ is the one-electron Dirac wave function, $A_{N}=1 / 2$ for equivalent electrons and $A_{N}=1 / \sqrt{2}$ for nonequivalent electrons. To account for the weak interaction we have to modify the wave function of the final state by admixing the close opposite-parity state:

$$
\begin{gathered}
\left|2^{1} S_{0}\right\rangle \rightarrow\left|2^{1} S_{0}\right\rangle+\frac{\left\langle 2^{3} P_{0}\left|H_{W}(1)+H_{W}(2)\right| 2^{1} S_{0}\right\rangle}{E_{2^{1} S_{0}}-E_{2^{3} P_{0}}}\left|2^{3} P_{0}\right\rangle, \\
\left|2^{3} P_{0}\right\rangle \rightarrow\left|2^{3} P_{0}\right\rangle+\frac{\left\langle 2^{1} S_{0}\left|H_{W}(1)+H_{W}(2)\right| 2^{3} P_{0}\right\rangle}{E_{2^{3} P_{0}}-E_{2^{1} S_{0}}}\left|2^{1} S_{0}\right\rangle .
\end{gathered}
$$

Here we have introduced the spin-independent part of the effective nuclear weak-interaction Hamiltonian [1]

$$
H_{W}=-\left(G_{F} / \sqrt{8}\right) Q_{W} \rho_{N}(r) \gamma_{5}
$$

where $G_{F}$ is the Fermi constant, $Q_{W} \approx-N+Z\left(1-4 \sin ^{2} \theta_{W}\right)$ is the weak charge of the nucleus, $\rho_{N}$ is the nuclear weak-charge density normalized to unity, and $\gamma_{5}$ is the Dirac matrix. For convenience, we rewrite formulas (5) -(66) as

$$
\begin{gathered}
\left|2^{1} S_{0}\right\rangle \rightarrow\left|2^{1} S_{0}\right\rangle+i \xi\left|2^{3} P_{0}\right\rangle, \\
\left|2^{3} P_{0}\right\rangle \rightarrow\left|2^{3} P_{0}\right\rangle+i \xi\left|2^{1} S_{0}\right\rangle,
\end{gathered}
$$


or in general

$$
\left|\Psi_{f}\right\rangle \rightarrow\left|\Psi_{f}\right\rangle+i \xi\left|\Psi_{\tilde{f}}\right\rangle
$$

where the parameter $\xi$ is given by

$$
\xi=\frac{G_{F}}{2 \sqrt{2}} \frac{Q_{W}}{E_{2^{1} S_{0}}-E_{2^{3} P_{0}}} \int_{0}^{\infty} d r r^{2} \rho_{N}(r)\left[g_{2 p_{1 / 2}} f_{2 s}-f_{2 p_{1 / 2}} g_{2 s}\right]
$$

with the large and small radial components of the Dirac wave function defined by

$$
\psi_{n \kappa m}(\mathbf{r})=\left(\begin{array}{c}
g_{n \kappa}(r) \Omega_{\kappa m}(\mathbf{n}) \\
i f_{n \kappa}(r) \Omega_{-\kappa m}(\mathbf{n})
\end{array}\right)
$$

With the PNC correction, the differential cross section is given by

$$
\begin{aligned}
\sigma=\frac{(2 \pi)^{4}}{v_{i}} & \mathbf{k}_{\mathbf{f}}^{2} \sum_{\boldsymbol{\epsilon}_{f}}\left\{\left|\sum_{M_{d}} \tau_{\gamma_{f}, f ; d} \frac{1}{E_{i}-E_{d}+i \Gamma_{d} / 2}\left\langle\Psi_{d}|I| \Psi_{i}\right\rangle+\tau_{\gamma_{f}, f ; i}\right|^{2}\right. \\
+ & 2 \Re\left[i \xi\left(\sum_{M_{d}} \tau_{\gamma_{f}, f ; d} \frac{1}{E_{i}-E_{d}+i \Gamma_{d} / 2}\left\langle\Psi_{d}|I| \Psi_{i}\right\rangle+\tau_{\gamma_{f}, f ; i}\right)\right. \\
& \left.\left.\times\left(\sum_{M_{d}^{\prime}} \tau_{\gamma_{f}, \tilde{f} ; d}^{*} \frac{1}{E_{i}-E_{d}-i \Gamma_{d} / 2}\left\langle\Psi_{d}|I| \Psi_{i}\right\rangle^{*}+\tau_{\gamma_{f}, \tilde{f} ; i}^{*}\right)\right]\right\} .
\end{aligned}
$$

To a good approximation, the RR amplitudes are calculated by formulas

$$
\begin{aligned}
& \tau_{\gamma_{f}, f ; i}=-\left\langle\Psi_{f}\left|R^{\dagger}(1)+R^{\dagger}(2)\right| \Psi_{i}\right\rangle, \\
& \tau_{\gamma_{f}, \tilde{f} ; i}=-\left\langle\Psi_{\tilde{f}}\left|R^{\dagger}(1)+R^{\dagger}(2)\right| \Psi_{i}\right\rangle
\end{aligned}
$$

where $R=e \alpha_{\mu} A^{\mu}=-e \boldsymbol{\alpha} \cdot \mathbf{A}$ is the transition operator acting on the electron variables, and

$$
\mathbf{A}(\mathbf{x})=\frac{\boldsymbol{\epsilon} \exp \left(i \mathbf{k}_{f} \cdot \mathbf{x}\right)}{\sqrt{2 k_{f}^{0}(2 \pi)^{3}}}
$$

is the wave function of the emitted photon. Calculations of the $\mathrm{RR}$ transition amplitude including the PNC effect were considered in detail in Ref. [16].

To enhance the PNC effect on the dielectronic recombination, one should consider the resonance with a state $d$ which decays to the final $f$ state via the M1 transition while the admixture of the $\tilde{f}$ state due to the weak interaction enables the E1 transition. As the simplest case, one can choose the state $d=\left(2 s_{1 / 2} 2 p_{1 / 2}\right)_{1}$, which decays into the state 


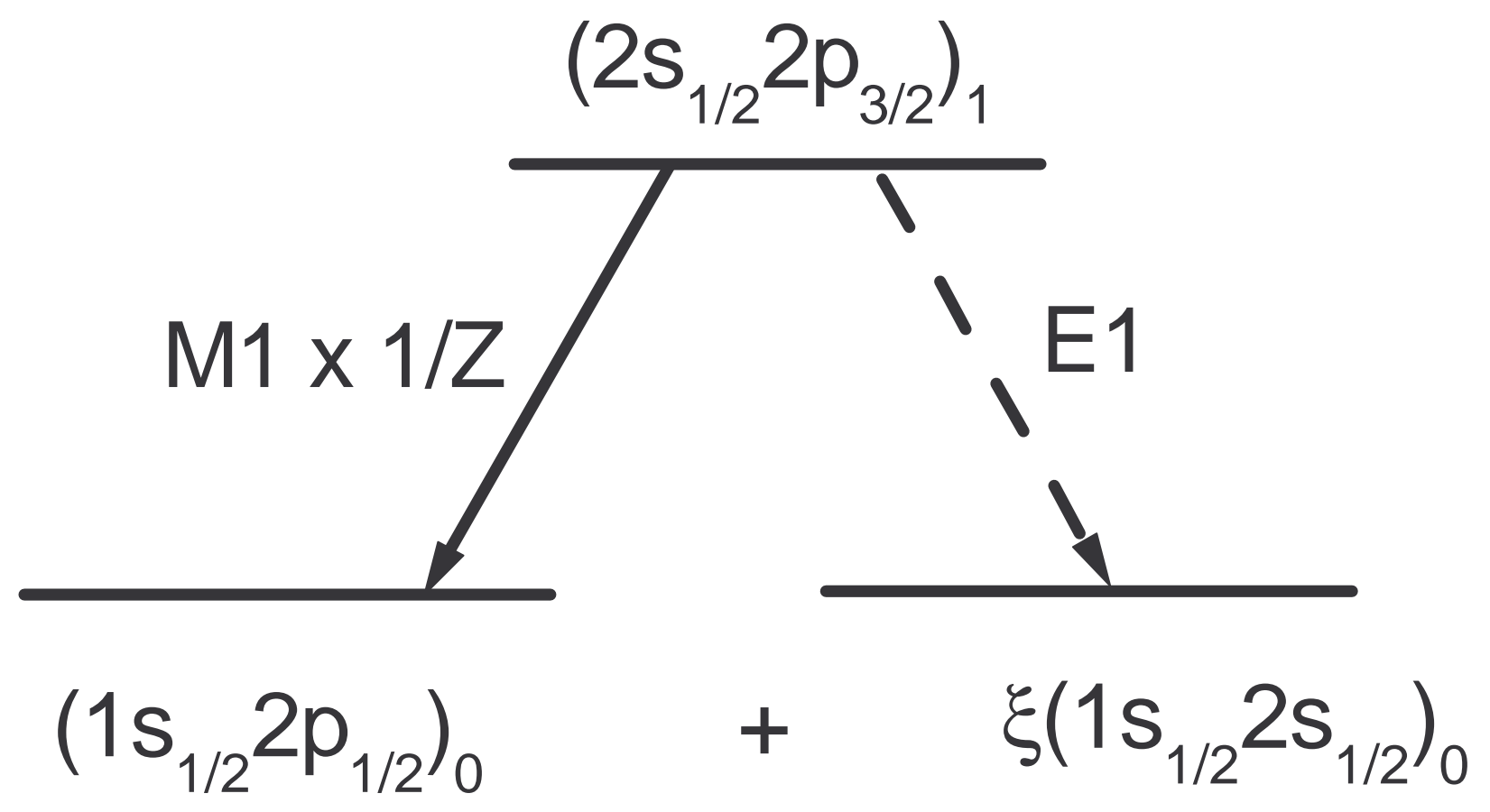

FIG. 1: The decay scheme of the $\left|\left(2 s_{1 / 2} 2 p_{3 / 2}\right)_{1}\right\rangle$ state into the $\left|2^{3} P_{0}\right\rangle+\xi\left|2^{1} S_{0}\right\rangle$ state. The main channel is the two-electron M1 transition, which is suppressed by a factor $1 / Z$, while the PNCmixing channel is the one-electron E1 transition.

$f=2^{3} P_{0}$ via the one-electron M1 transition, whereas the PNC mixing transition is the one-electron E1 transition. In this case the $\tau_{\gamma_{f}, f ; d}$ and $\tau_{\gamma_{f}, \tilde{f} ; d}$ amplitudes can be calculated within the lowest-order approximation by formulas which are similar to (14)-(15),

$$
\begin{gathered}
\tau_{\gamma_{f}, f ; d}=-\left\langle\Psi_{f}\left|R^{\dagger}(1)+R^{\dagger}(2)\right| \Psi_{d}\right\rangle, \\
\tau_{\gamma_{f}, \tilde{f} ; d}=-\left\langle\Psi_{\tilde{f}}\left|R^{\dagger}(1)+R^{\dagger}(2)\right| \Psi_{d}\right\rangle .
\end{gathered}
$$

A bigger enhancement of the PNC effect on the dielectronic recombination should occur in a situation where the doubly excited state $d$ decays into the final $f$ state via the two-electron M1 transition, which is additionally suppressed by a factor $1 / Z$ compared to the one-electron M1 transition, while the PNC mixing transition is the one-electron E1 transition. As an example, we can consider $d=\left(2 s_{1 / 2} 2 p_{3 / 2}\right)_{1}$. The related decay scheme is shown in Fig. 团 where the solid line displays the main channel that is the two-electron M1 transition and the dashed line indicates the PNC-mixing channel that is the one-electron E1 transition. Since the $\mathrm{E} 1$ transition is very strong compared to the $\mathrm{M} 1 \times 1 / Z$ transition, the admixture of the $2^{1} S_{0}$ state to the $2^{3} P_{0}$ state due to the weak interaction should be most pronounced in the 
DR contribution.

The two-electron transition amplitude can be evaluated by perturbation theory [22, 23]. Let the two-electron states $d$ and $f$ are defined by sets of one-electron states $\left(d_{1}, d_{2}\right)$ and $\left(f_{1}, f_{2}\right)$, respectively. For the case under consideration (all four states $d_{1}, d_{2}, f_{1}$ and $f_{2}$ are different) the two-electron transition amplitude is given by (for more details see Ref. [23])

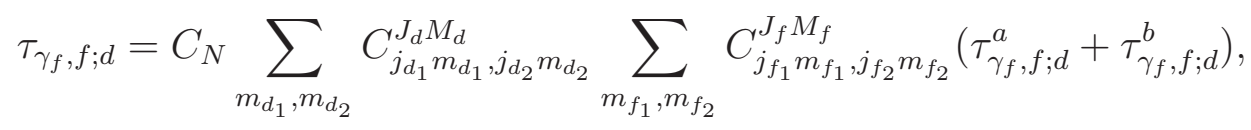

where

$$
\begin{aligned}
& \tau_{\gamma_{f}, f ; d}^{a}=-\sum_{\mathcal{P}}(-1)^{\mathcal{P}}\left\{\sum_{n}\left\langle\mathcal{P} f_{1}\left|e \alpha_{\mu} A^{\mu *}\right| n\right\rangle \frac{1}{E_{d}^{(0)}-\varepsilon_{\mathcal{P} f_{2}}-\varepsilon_{n}}\left\langle n \mathcal{P} f_{2}\left|I\left(\varepsilon_{\mathcal{P} f_{2}}-\varepsilon_{d_{2}}\right)\right| d_{1} d_{2}\right\rangle\right. \\
&+\left.\sum_{n}\left\langle\mathcal{P} f_{2}\left|e \alpha_{\mu} A^{\mu *}\right| n\right\rangle \frac{1}{E_{d}^{(0)}-\varepsilon_{\mathcal{P} f_{1}}-\varepsilon_{n}}\left\langle\mathcal{P} f_{1} n\left|I\left(\varepsilon_{\mathcal{P} f_{1}}-\varepsilon_{d_{1}}\right)\right| d_{1} d_{2}\right\rangle\right\}, \\
& \tau_{\gamma_{f}, f ; d}^{b}=-\sum_{\mathcal{P}}(-1)^{\mathcal{P}}\left\{\sum_{n}\left\langle\mathcal{P} f_{1} \mathcal{P} f_{2}\left|I\left(\varepsilon_{\mathcal{P} f_{2}}-\varepsilon_{d_{2}}\right)\right| n d_{2}\right\rangle \frac{1}{E_{f}^{(0)}-\varepsilon_{d_{2}}-\varepsilon_{n}}\left\langle n\left|e \alpha_{\mu} A^{\mu *}\right| d_{1}\right\rangle\right. \\
&\left.+\sum_{n}\left\langle\mathcal{P} f_{1} \mathcal{P} f_{2}\left|I\left(\varepsilon_{\mathcal{P} f_{1}}-\varepsilon_{d_{1}}\right)\right| d_{1} n\right\rangle \frac{1}{E_{f}^{(0)}-\varepsilon_{d_{1}}-\varepsilon_{n}}\left\langle n\left|e \alpha_{\mu} A^{\mu *}\right| d_{2}\right\rangle\right\},
\end{aligned}
$$

$C_{N}$ is the normalization factor which is equal to 1 in case of non-equivalent electrons in both

initial and final states, $E_{f}^{(0)}=\varepsilon_{f_{1}}+\varepsilon_{f_{2}}, E_{d}^{(0)}=\varepsilon_{d_{1}}+\varepsilon_{d_{2}}$, and $\varepsilon_{n}$ is the one-electron Dirac energy.

So far we have assumed that the intermediate and final states are well isolated single levels. This approximation, being valid for the final states, can be incorrect for the intermediate states which have rather large widths. To account for quasidegeneracy of the intermediate states within the rigorous QED approach, one can use the methods described in Refs. [22, 24]. For our purposes, however, it is sufficient to use a simple replacement of the expression (13) by one which contains a sum over all close-lying intermediate states and account for the mixing of the states having the same symmetry.

\section{NUMERICAL RESULTS AND DISCUSSION}

The most promising situation for observing the PNC effect in the process under consideration occurs for gadolinium $(Z=64)$ and thorium $(Z=90)$. The energy difference 
between the levels $2^{1} S_{0}$ and $2^{3} P_{0}$ amounts to $-0.023(74)$ for gadolinium and to $0.44(40) \mathrm{eV}$ for thorium [17, 25, 26]. As in our previous papers [16, 17], to estimate the PNC effect we use $0.44 \mathrm{eV}$ and $0.074 \mathrm{eV}$ for the $2^{3} P_{0}-2^{1} S_{0}$ energy difference in cases of Th and Gd, respectively.

Let us consider the requirement that should be imposed on the luminosity $L$, provided the PNC effect is measured to a relative accuracy $\eta$. Denoting by $\sigma_{+}$and $\sigma_{-}$the differential cross sections for the positive and negative spin projection of the incident electron onto the electron momentum, one can derive [14, 16]

$$
L>L_{0}=\frac{\sigma_{+}+\sigma_{-}+2 \sigma_{\mathrm{b}}}{\left(\sigma_{+}-\sigma_{-}\right)^{2} \eta^{2} T}
$$

where $\sigma_{\mathrm{b}}$ is the background magnitude and $T$ is the acquisition time. In our calculations we set $T$ equal to two weeks and neglect the background signal. We have studied resonance recombination processes which correspond to the following DR channels (the admixture due to the weak interaction is implied):
1) $\bar{e}+1 s \rightarrow\left(2 s_{1 / 2} 2 p_{1 / 2}\right)_{1} \rightarrow\left(1 s_{1 / 2} 2 p_{1 / 2}\right)_{0}+\gamma$
2) $\bar{e}+1 s \rightarrow\left(2 s_{1 / 2} 2 p_{3 / 2}\right)_{1} \rightarrow\left(1 s_{1 / 2} 2 p_{1 / 2}\right)_{0}+\gamma$
3) $\bar{e}+1 s \rightarrow\left(2 p_{1 / 2} 2 p_{3 / 2}\right)_{1} \rightarrow\left(1 s_{1 / 2} 2 s_{1 / 2}\right)_{0}+\gamma$
4) $\bar{e}+1 s \rightarrow\left(2 s_{1 / 2} 3 p_{1 / 2}\right)_{1} \rightarrow\left(1 s_{1 / 2} 2 p_{1 / 2}\right)_{0}+\gamma$
5) $\bar{e}+1 s \rightarrow\left(2 s_{1 / 2} 3 p_{3 / 2}\right)_{1} \rightarrow\left(1 s_{1 / 2} 2 p_{1 / 2}\right)_{0}+\gamma$

The first process is the only one in which the M1 transition from the resonance state is not suppressed by a factor $1 / Z$. All other processes include the two-electron M1 transition to the final state while the PNC-mixing channel is the one-electron E1 transition. For all processes we evaluated the differential cross section (13) as a function of the photon emission angle $(\theta)$ with respect to the incident electron momentum. The calculations of the Dirac wave functions that enter the formulas were performed using the RADIAL package [27] and the dual-kinetic-balance basis set method [28] with the basis functions constructed from B-splines [29].

Table I presents numerical results for the differential cross section in case of thorium at the angles $\theta$ corresponding to the minimum values of the luminosity. Table II presents the related results for gadolinium. We denote the cross section without the PNC effect as $\sigma_{0}=\left(\sigma_{+}+\sigma_{-}\right) / 2$ and the PNC contribution as $\sigma_{\mathrm{PNC}}=\left(\sigma_{+}-\sigma_{-}\right) / 2 . N$ is the process number in the list above. As can be seen from the tables, the most favourable are the 1-st, 2-nd, 
TABLE I: Differential cross section for resonance recombination of a polarized electron with H-like thorium at the photon emission angle $\theta$ corresponding to the minimum value of the luminosity $L_{0}$ which is defined by Eq. (22) at $T=2$ weeks. $\sigma_{0}$ is the cross section without the PNC effect and $\sigma_{\mathrm{PNC}}$ is the PNC contribution. Results are presented in ascending order of the luminosity $L_{0}, N$ is the process number defined in the text.

\begin{tabular}{cccccc}
\hline$N \varepsilon_{i}[\mathrm{keV}]$ & $\theta[\mathrm{deg}]$ & $L_{0}\left[\mathrm{~cm}^{-2} \mathrm{~s}^{-1}\right]$ & $\sigma_{0}[\mathrm{barn}]$ & $\sigma_{\mathrm{PNC}}[\mathrm{barn}]$ \\
\hline 1 & 60.91 & 0 & $1.2 \times 10^{30}$ & 1.076 & $6.1 \times 10^{-5}$ \\
2 & 65.04 & 56 & $1.9 \times 10^{30}$ & 1.247 & $-5.2 \times 10^{-5}$ \\
4 & 79.21 & 0 & $1.9 \times 10^{30}$ & 0.429 & $3.0 \times 10^{-5}$ \\
5 & 80.41 & 53 & $3.2 \times 10^{30}$ & 0.779 & $-3.2 \times 10^{-5}$ \\
3 & 64.96 & 43 & $2.0 \times 10^{31}$ & 0.287 & $7.7 \times 10^{-6}$ \\
\hline
\end{tabular}

and 4 -th processes. In all these cases the final state is $\left|2^{3} P_{0}\right\rangle$ with an admixture of $\left|2^{1} S_{0}\right\rangle$ due to the weak interaction. In Figs. 2, 3, and 4 we display the values $\sigma_{\mathrm{PNC}}^{2} / \sigma_{0} \sim 1 / L_{0}$ as functions of $\theta$ for 1-st, 2-nd, and 4-th processes. According to the tables, the PNC asymmetry of the cross section does exceed $0.01 \%$. It should be stressed, however, that the PNC asymmetry on some of the DR contributions, taken separately, is very large and for the resonance DR into the $\left(2 p_{1 / 2} 2 p_{3 / 2}\right)_{1}$ state amounts to about $12 \%$ in case of thorium. This is extremely large value for atomic PNC effects. But, unfortunately, this large value is strongly masked by the RR and non-resonance DR contributions. This fact, together with current restrictions on the experimental resolution (see the related discussion in Ref. [16]), make practical realization of such an experiment rather problematic. We think, however, that the calculations performed will help us to search for more realistic scenarios to observe the PNC effect in resonance scattering processes with heavy few-electron ions.

In summary, we have studied the PNC effect on the cross section of resonance recombination of polarized electrons with H-like thorium and gadolinium, where the PNC effect is strongly enhanced due quasidegeneracy of the opposite-parity $2^{1} S_{0}$ and $2^{3} P_{0}$ states. The calculations were performed for different intermediate doubly excited states, which can decay into one of the $2^{1} S_{0}$ or $2^{3} P_{0}$ states via one-photon emission. It was found that the most promising situation occurs when the incident electron energy is chosen to be in resonance with the $\left(2 s_{1 / 2} 2 p_{1 / 2}\right)_{1}$ state. We hope that this work will stimulate further efforts for 
TABLE II: Differential cross section for resonance recombination of a polarized electron with H-like gadolinium at the photon emission angle $\theta$ corresponding to the minimum value of the luminosity $L_{0}$ which is defined by Eq. (22) at $T=2$ weeks. $\sigma_{0}$ is the cross section without the PNC effect and $\sigma_{\mathrm{PNC}}$ is the PNC contribution. Results are presented in ascending order of the luminosity $L_{0}$, $N$ is the process number defined in the text.

\begin{tabular}{cccccc}
\hline$N \varepsilon_{i}[\mathrm{keV}]$ & $\theta[\mathrm{deg}]$ & $L_{0}\left[\mathrm{~cm}^{-2} \mathrm{~s}^{-1}\right]$ & $\sigma_{0}[\mathrm{barn}]$ & $\sigma_{\mathrm{PNC}}[\mathrm{barn}]$ \\
\hline 2 & 30.27 & 66 & $4.3 \times 10^{30}$ & 0.996 & $3.1 \times 10^{-5}$ \\
5 & 37.93 & 71 & $7.5 \times 10^{30}$ & 0.568 & $1.8 \times 10^{-5}$ \\
1 & 29.36 & 0 & $7.7 \times 10^{30}$ & 0.467 & $-1.6 \times 10^{-5}$ \\
4 & 37.67 & 0 & $2.1 \times 10^{31}$ & 0.218 & $-6.6 \times 10^{-6}$ \\
3 & 30.24 & 40 & $1.9 \times 10^{32}$ & 0.331 & $-2.7 \times 10^{-6}$ \\
\hline
\end{tabular}

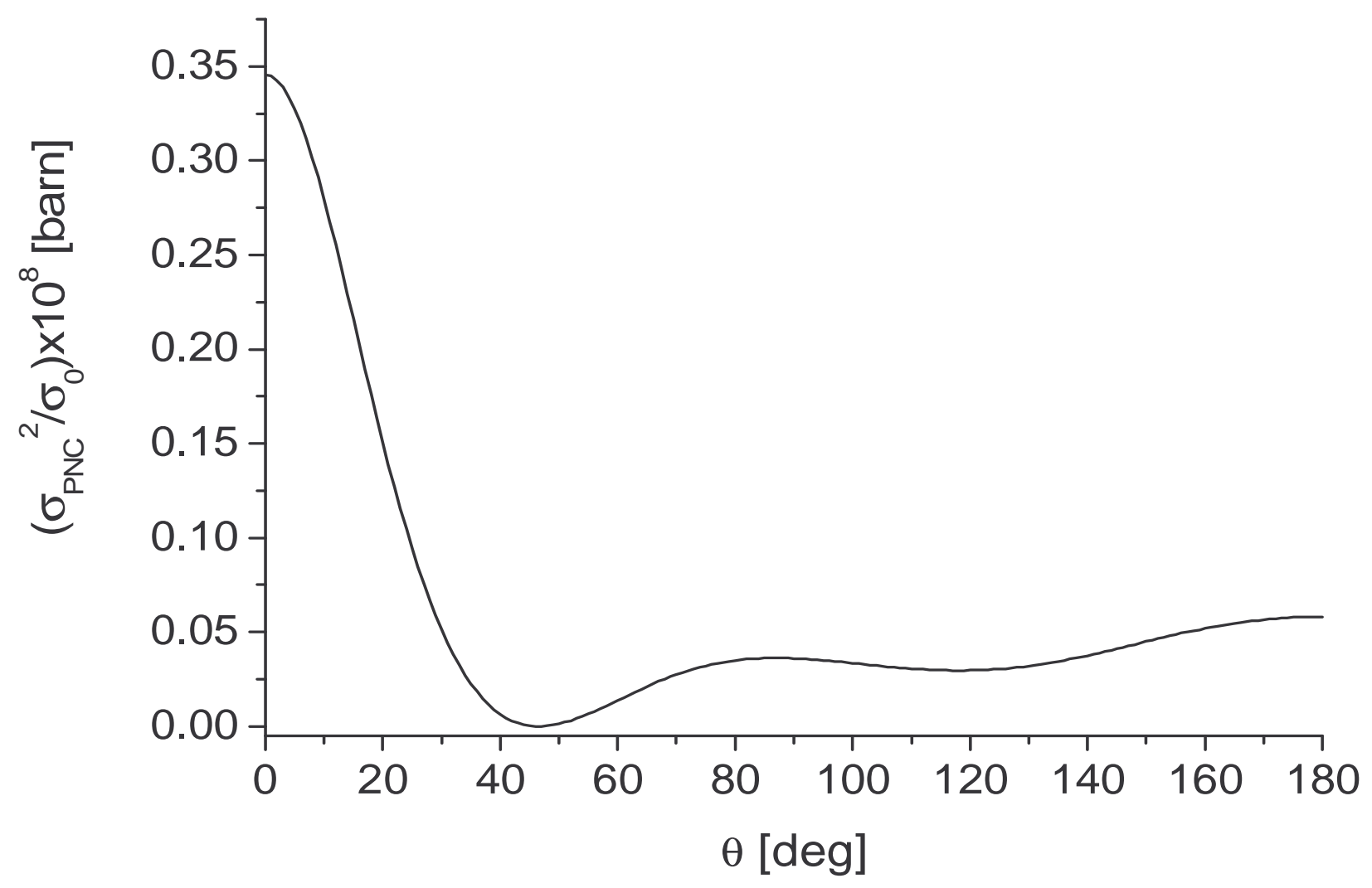

FIG. 2: The value $\sigma_{\mathrm{PNC}}^{2} / \sigma_{0} \sim 1 / L_{0}$ as a function of photon emission angle $\theta$ for the resonance recombination into the $2^{3} P_{0}$ state of He-like thorium with intermediate state $\left(2 s_{1 / 2} 2 p_{1 / 2}\right)_{1}$, 1 -st process. 


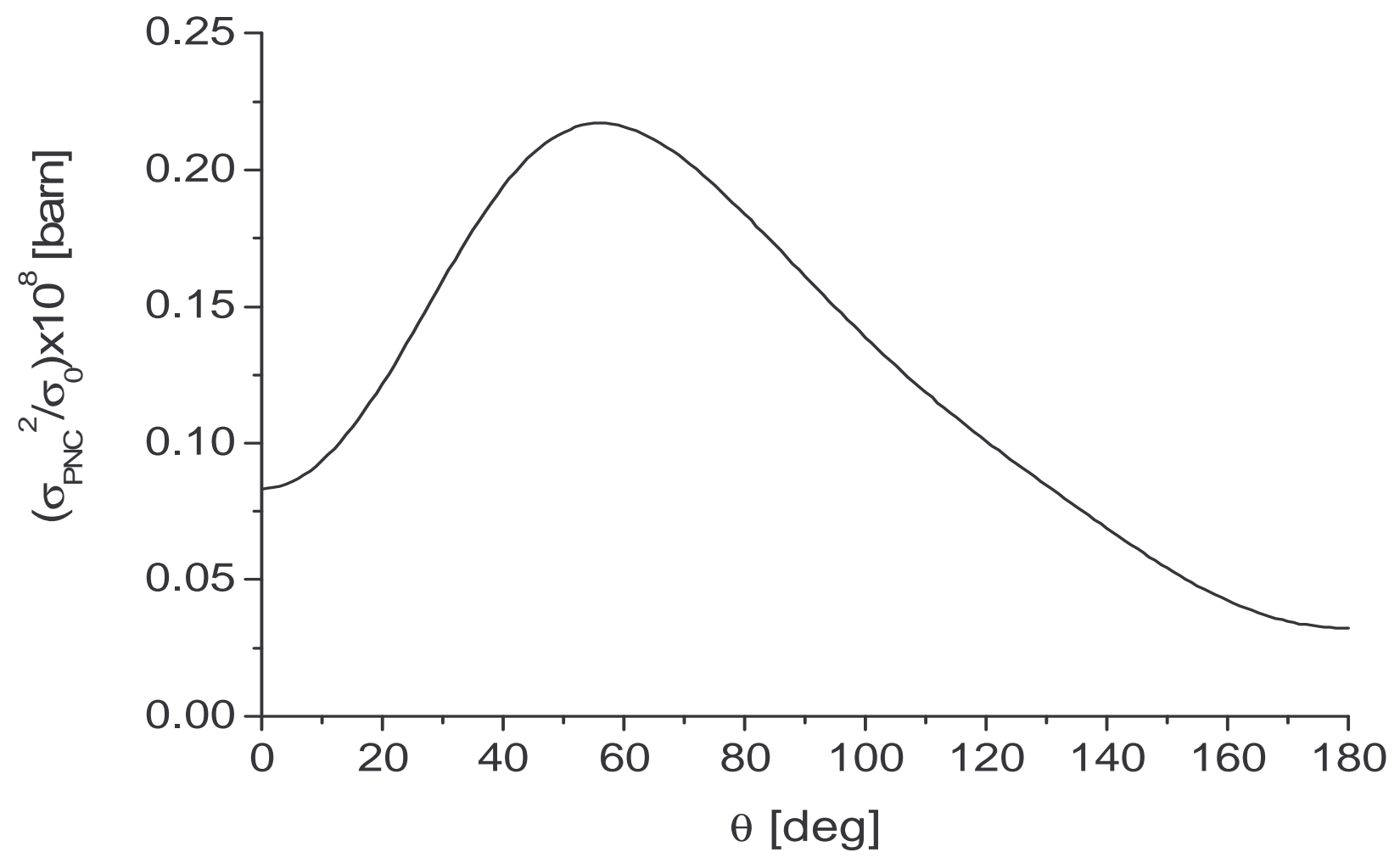

FIG. 3: The value $\sigma_{\mathrm{PNC}}^{2} / \sigma_{0} \sim 1 / L_{0}$ as a function of photon emission angle $\theta$ for the resonance recombination into the $2^{3} P_{0}$ state of He-like thorium with intermediate state $\left(2 s_{1 / 2} 2 p_{3 / 2}\right)_{1}, 2$-nd process.

studying the PNC effects in resonance scattering processes with heavy ions.

\section{Acknowledgments}

This work was supported by DFG (Grants No. PL 254/7-1 and VO 1707/1-1), by RFBR (Grant No. 10-02-00450), by GSI, by DAAD, by the Ministry of Education and Science of Russian Federation (Program "Scientific and pedagogical specialists for innovative Russia", Grant No. P1334). The work of A.V.M. and V.A.Z. was also supported by the "Dynasty" foundation.

[1] I.B. Khriplovich, Parity Nonconservation in Atomic Phenomena, (Gordon and Breach, London, 1991). 


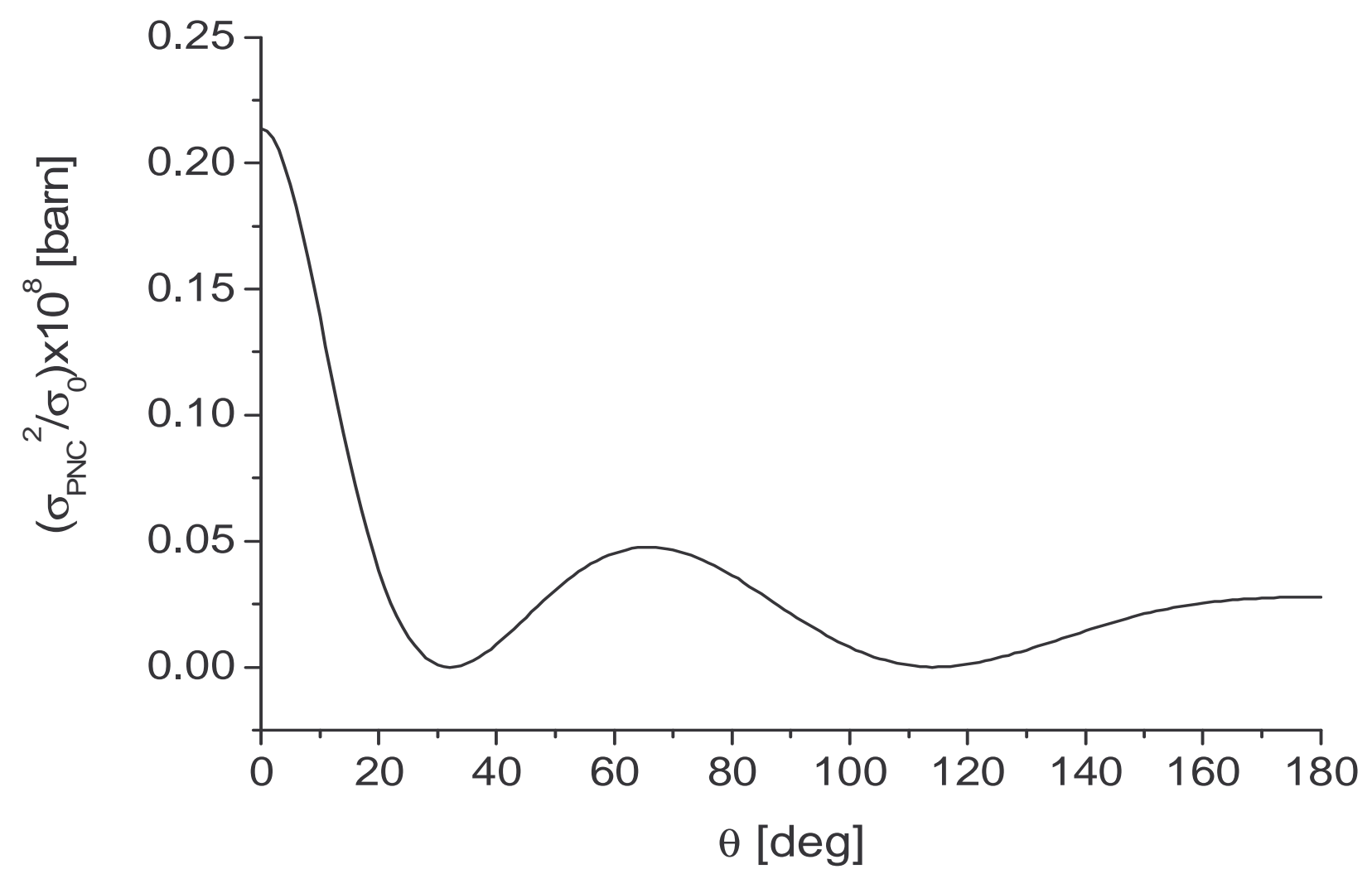

FIG. 4: The value $\sigma_{\mathrm{PNC}}^{2} / \sigma_{0} \sim 1 / L_{0}$ as a function of photon emission angle $\theta$ for the resonance recombination into the $2^{3} P_{0}$ state of He-like thorium with intermediate state $\left(2 s_{1 / 2} 3 p_{1 / 2}\right)_{1}, 4$-th process.

[2] I.B. Khriplovich, Phys. Scr. T 112, 52 (2004).

[3] J.S.M. Ginges and V.V. Flambaum, Phys. Rep. 397, 63 (2004).

[4] V.M. Shabaev, K. Pachucki, I.I. Tupitsyn, and V.A. Yerokhin, Phys. Rev. Lett. 94, 213002 (2005).

[5] S. G. Porsev, K. Beloy and A. Derevianko, Phys. Rev. Lett., 102, 181601 (2009).

[6] V. G. Gorshkov and L. N. Labzowsky, Zh. Eksp. Teor. Fiz. Pis'ma 19, 768 (1974) [JETP Lett. 19, 394 (1974)]; Zh. Eksp. Teor. Fiz. 69, 1141 (1975) [Sov. Phys. JETP 42, 581 (1975)].

[7] A. Schäfer, G. Soff, P. Indelicato, B. Müller, and W. Greiner, Phys. Rev. A 40, 7362 (1989).

[8] G. von Oppen, Z. Phys. D 21, 181 (1991).

[9] V. V. Karasiev, L. N. Labzowsky, and A. V. Nefiodov, Phys. Lett. A 172, 62 (1992).

[10] M. S. Pindzola, Phys. Rev. A 47, 4856 (1993).

[11] R. W. Dunford, Phys. Rev. A 54, 3820 (1996). 
[12] M. Zolotorev and D. Budker, Phys. Rev. Lett. 78, 4717 (1997).

[13] L. N. Labzowsky, A. V. Nefiodov, G. Plunien, G. Soff, R. Marrus, and D. Liesen, Phys. Rev. A 63, 054105 (2001).

[14] G. F. Gribakin, F. J. Currell, M. G. Kozlov, A. I. Mikhailov, Phys. Rev. A 72, 032109 (2005); 80, 049901(E) (2009); arXiv:physics/0504129.

[15] L. Labzowsky and A. Prozorov, J. Phys.: Conf. Ser. 72, 012010 (2007).

[16] A. V. Maiorova, O. I. Pavlova, V. M. Shabaev, C. Kozhuharov, G. Plunien, and T. Stöhlker, J. Phys. B 42, 205002 (2009).

[17] V. M. Shabaev, A. V. Volotka, C. Kozhuharov, G. Plunien, and T. Stöhlker, Phys. Rev. A 81, $052102(2010)$.

[18] F. Ferro, A. Artemyev, T. Stöhlker, and A. Surzhykov, Phys. Rev. A 81, 062503 (2010).

[19] F. Ferro, A. Surzhykov, and T. Stöhlker, Phys. Rev. A 83, 052518 (2011).

[20] V. V. Karasiov, L. N. Labzowsky, A. V. Nefiodov, and V. M. Shabaev, Phys. Lett. A 161, 453 (1992).

[21] V. M. Shabaev, Phys. Rev. A 50, 4521 (1994).

[22] V. M. Shabaev, Phys. Rep. 356, 119 (2002).

[23] P. Indelicato, V. M. Shabaev and A. V. Volotka, Phys. Rev. A 69, 062506 (2004).

[24] O. Yu. Andreev, L. N. Labzowsky, G. Plunien, D. A. Solovyev, Phys. Rep. 455, 135 (2008).

[25] A.N. Artemyev, V.M. Shabaev, V.A. Yerokhin, G. Plunien, and G. Soff, Phys. Rev. A 71, $062104(2005)$.

[26] Y.S. Kozhedub, O.V. Andreev, V.M. Shabaev, I.I. Tupitsyn, C. Brandau, C. Kozhuharov, G. Plunien, T. Stöhlker, Phys. Rev. A 77, 032501 (2008).

[27] F. Salvat, J. M. Fernández-Varea, and W. Williamson Jr., Comput. Phys. Commun. 90, 151 (1995).

[28] V.M. Shabaev, I.I. Tupitsyn, V.A. Yerokhin, G. Plunien, and G. Soff, Phys. Rev. Lett. 93, 130405 (2004).

[29] J. Sapirstein and W. R. Johnson, J. Phys. B 29, 5213 (1996). 